\title{
Characteristics of Very Fast Transient Currents in Ultra High-Voltage Power System with Hybrid Reactive Power Compensation
}

\author{
Hongshun Liu ${ }^{\mathrm{a}}$, Taiyu Chen ${ }^{\mathrm{a}}$, Qiuqin Sun ${ }^{\mathrm{b}}$, Mingming Han ${ }^{\mathrm{c}}$, Qingquan $\mathrm{Li}^{\mathrm{a}}$, and Wah.Hoon.Siew ${ }^{\mathrm{d}}$
}

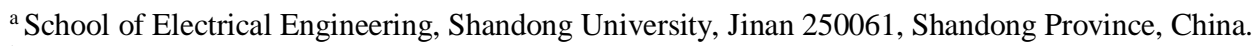

${ }^{\mathrm{b}}$ College of Electrical and Information Engineering, Hunan University, Changsha, China.

${ }^{c}$ State Grid Shandong Electric Power Research Institute, Jinan, China.

${ }^{\mathrm{d}}$ Department of Electronic \& Electrical Engineering, the University of Strathclyde, Glasgow, Scotland, United Kingdom.

*Corresponding author (E-mail: sunqq@hnu.edu.cn)
\end{abstract}

Hybrid reactive power compensation (HRPC) consists of a stepped controlled shunt reactor (SCSR) and a series compensation (SC), which will find applications in future ultra high-voltage (UHV) power grids to resolve the problems due to the frequent change of reactive power and bulk power transmission. However, very fast transient currents (VFTCs) are inevitably generated during switching, which would lead to insulation breakdown. In the present work, we first develop the equivalent model for HRPC, following which we deduce the expression of VFTCs in the time domain by using an inverse Laplace transform. The analysis indicates that the amplitude and frequency of VFTCs are both affected by the capacitance of the SCSR and of the SC, as well as the line length, stray capacitance, etc. The oscillating frequency, peak, and amplitude of the main frequency of the VFTCs in the substation can be modified by adjusting the silicon-controlled rectifiers in the SCSR when the disconnecting switch in gas-insulated switchgear is switched on. When the disconnecting switch in the SC is switched on, the VFTC oscillation frequency in SCSR decreases with increasing stray capacitance of SC, but the frequency and peak of the VFTC remains quite large. Increasing the line length between the SC and the SCSR suppresses the VFTC in UHV power systems. These results lay the foundation for developing HRPC methods to suppress VFTCs in UHV substations.

Index Terms - amplitude-frequency characteristics; hybrid reactive power compensation; Inverse Laplace Transform; stray capacitance; very fast transient current

\section{INTRODUCTION}

Long-distance bulk-capacity low-loss transmission can be realized in an ultra high-voltage (UHV) AC power grid. In addition, UHV systems offer excellent performance, such as the optimal allocation of resources and the prevention of haze fog $[1,2]$. The large-scale construction of UHV power systems is becoming the new norm in China's electric power industry, which is an indication of China's current prosperity [3, 4]. However, in the future, power transmission will significantly increase and reactive power will undergo frequent changes. The security and stability of the UHV synchronous power system will face new challenges, which are manifested in two main ways: (1) the rapid growth in demand for power transmission cannot be satisfied due to the impedance characteristics and the stability limitation of power systems [5], and (2) the increasing exchange of power between different regional grids amplifies the dynamic variations in reactive power in transmission lines and increases the amplitude and frequency of voltage variations, which makes it difficult to adjust the voltage with traditional reactive devices (only fixed shunt reactors) [6]. Hybrid reactive power compensation (HRPC), which consists of series compensation (SC) and a stepped controlled shunt reactor (SCSR) can balance the growth of active power transmission and the adjustment of reactive power [7-10]. This solution is ideal to overcome the disadvantages of traditional simple reactive compensation mode, which can increase the flexibility and reliability of reactive compensation of UHV systems. Thus, the HRPC technique will see use in China's future UHV transmission system.
Very fast transient currents (VFTCs) are generated when the disconnecting switch (DS) is operated and these currents can cause insulation breakdown in the associated power equipment [11]. In particular, a proper understanding of the characteristics of VFTCs is of great importance for protecting gas-insulated switchgear (GIS). Based on a $252 \mathrm{kV}$ GIS experimental investigation, an arc model to simulate very fast transient overvoltage (VFTO) was proposed [12]. This work revealed the time dependence of the arc resistance. The peak magnitude of the VFTC and the dominant frequency content at various locations were also computed in a $245 \mathrm{kV}$ GIS for different switching operations and substation configurations [13]. In other work, a derivative Gaussian current source and a short-time sinusoidal current source were used to evaluate the effectiveness of the shielding of gas-insulated bus ducts against transient electromagnetic fields generated in a GIS during switching [14].

However, given the high voltage and bulk power capacity of GIS UHV substations, the problems stemming from VFTCs are more challenging. The formation mechanism, key factors, and techniques to suppress VFTCs and VFTOs were extensively studied over the past decade $[15,16]$. Reference [17] proposed a method to compute the voltage and current of key points along the transmission line, and Ref. [18] studied the three-dimensional electrostatic field of DSs by using the finite-element method and the distribution of electric field of the GIS. Other studies demonstrate that the amplitude-frequency characteristics of VFTOs are influenced by the substation or switching-station layout, as discussed in Refs. [19, 20]. In addition, Ref. [21] analyzed the adverse effect 
of VFTOs on power equipment and the advantages and disadvantages of various suppression techniques [21]. By simplifying the DS arc resistance using a hyperbolic resistance model, Refs. [22, 23] analyzed the frequency-domain solution of bus-bar voltage and how switch arc affects VFTOs and presented a series of experimental data, including the permeability of magnetic materials in the complex-frequency magnetic domain, to support power systems in practical applications. Installing an UHV substation with HRPC means that the substation will be exposed to a new type of VFTC. The amplitude and frequency characteristics of VFTCs are influenced by the SCSR and the SC, the line length, and the stray capacitance of the SC. However, these amplitude-frequency characteristics of the VFTC have yet to be studied in connection with UHV systems with HRPC. Thus, studying how HRPC affects the characteristics of VFTCs in GIS UHV substations is critically important.

This work is organized as follows: Section 2 presents the principle and equivalent model of HRPC. Section 3 uses an inverse Laplace transform to derive time-domain expression for VFTCs. Finally, Section 4 uses the model developed in Section 3 to study the characteristics of the VFTCs generated by the DS in GISs and the operation of DS in SC. The results reported herein provide a theoretical foundation for optimizing HRPC design and methods to suppress VFTCs in UHV substations.

\section{PRINCIPLE OF HYBRID REACTIVE-POWER COMPENSATION}

HRPC generally consists of SC combined with SCSR [24-26]. The former consists of a capacitor bank, a zinc-oxide arrester, a spark gap, a bypass breaker, and a damping device, as depicted in Fig. 1(a). During normal operation, only the capacitor bank is put into service. The arrester normally serves to protect the capacitor bank, whereas the spark gap is used to protect the arrester from overheating. The bypass disconnector and the two disconnecting switches are indispensable for system maintenance and scheduling. The bypass breaker is used to facilitate the deionization of the spark gap under fault conditions. The damping device restricts the discharge current and prevents equipment failure. The zinc-oxide arrester sustains the voltage across the capacitor with a safety margin of 2.3 times the nominal voltage across the capacitor bank. The zinc-oxide arrester bypasses the capacitor bank when the overvoltage across the capacitor bank exceeds the above sustained voltage. The spark-gap trigger voltage is 2.4 times the nominal voltage across the capacitor bank. Usually, the spark gap is triggered $1 \mathrm{~ms}$ after the zinc-oxide arrester is used. Subsequently, the bypass breaker is switched on after the over-current signal of the relay protection with about $30 \mathrm{~ms}$ delay due to its mechanical-action time. Thus bypass breaker usually closes about $40 \mathrm{~ms}$ after the overvoltage across the capacitor bank.

The SCSR consists of a high-impedance transformer, a set of series reactors, mechanical switches, and silicon-controlled rectifiers. The typical stepped capacity of a SCSR is: $25 \%, 50 \%$, $75 \%$, and $100 \%$, as shown in Fig. 1(b). The inductive reactive power can be adjusted by the silicon-controlled rectifier. The secondary line winding of the SCSR is connected to a set of series reactors, the number of which is controlled by thyristors and mechanical switches. The thyristors provide fast regulation of the capacity of controlled shunt reactor, whereas the mechanical switches are used in bypass-mode during steady-state operation to allow the thyristors to cool.

The strategy to control the compensation degree of the SCSR is as follows: When the SCSR detects a change in the system voltage, the stepped capacity of SCSR should be rapidly adjusted. If the stepped capacity of SCSR should be decreased, one or more thyristors and the corresponding mechanical switches will be closed to increase the number of connected series reactors. After the mechanical switches are closed, the corresponding thyristors are blocked. If the stepped capacity of the SCSR should be increased, one or more of the mechanical switches will be opened to decrease the number of connected series reactors.

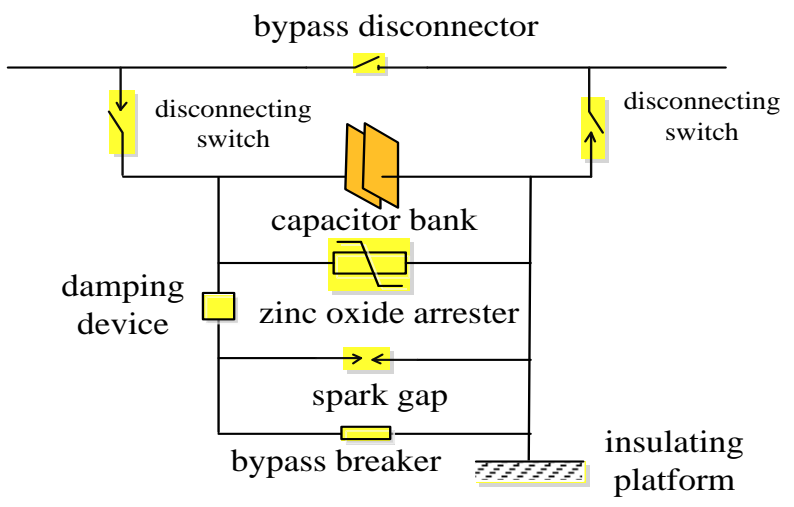

(a)

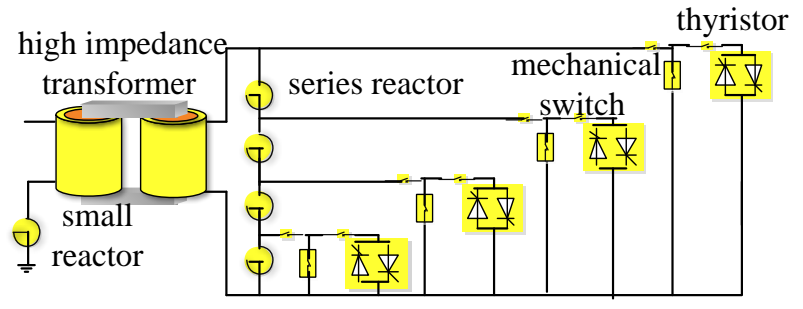

(b)

FIG. 1. Schematic diagram of hybrid reactive-power compensation: (a) series compensation, (b) stepped controlled shunt reactor.

No model of HRPC including the effect of VFTCs yet exists. The CIGRE report provides a lumped-parameter model of a SC and a SCSR (see Fig. 2) [27].

Figure 2(a) shows the SC model. Considering the real operating conditions, the two DSs at both ends of the capacitor bank are closed when a VFTC occurs. Thus, these two DSs are not modeled as capacitors. In Fig. 2(a), $C_{\mathrm{D}}, C_{\mathrm{C}}, C_{\mathrm{M}}, C_{\mathrm{H}}, C_{\mathrm{L}}$, and $C_{\mathrm{S}}$ represent the capacitance of the bypass switch, capacitor bank, arrester, spark gap, damping device, and bypass breaker, respectively. Note that $C_{\mathrm{C}}$ is much larger than the other capacitances. Figure 2(b) shows the model of a SCSR, where $C_{1}$ and $C_{2}$ represent the equivalent capacitance on the primary side and secondary side of the SCSR, respectively. $C_{12}$ is the 
equivalent transfer capacitance between the primary side and the secondary side of the SCSR.

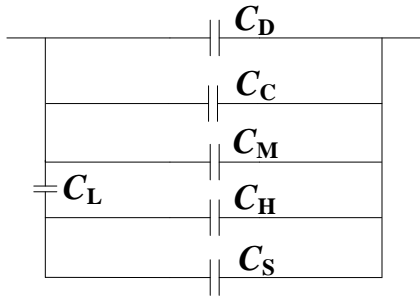

(a)

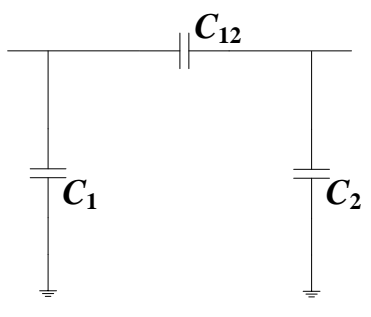

(b)
FIG. 2. Equivalent model of hybrid reactive power compensation: (a) series compensation, (b) stepped controlled shunt reactor.

\section{Theoretical Analysis of Very Fast Transient Current}

In very fast transient analysis, equivalent models of transformers and circuit breakers are often represented by capacitors. Other power equipment such as current transformers, insulators, and grounding switches, can be modeled by bus-bars of a certain length, assuming no corona and omitting line parallel conductance. Figure 3 shows a model of an UHV substation.

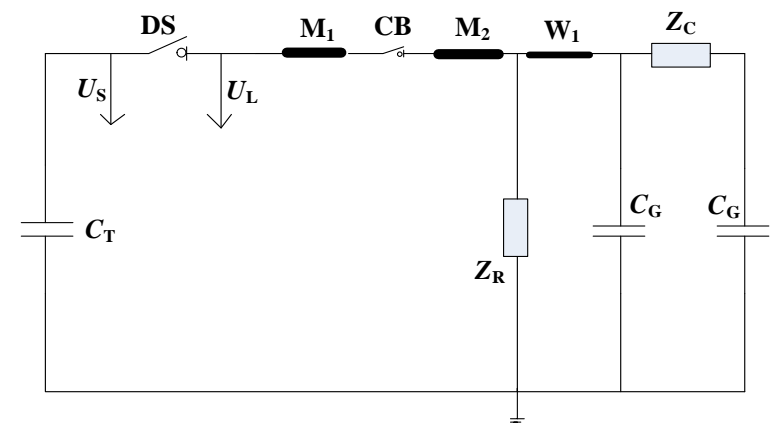

FIG. 3. Simplified model of ultrahigh voltage system.

In Fig. 3,

$C_{\mathrm{T}}$ is the capacitance of the power transformer;

$U_{\mathrm{S}}$ is the power supply;

$D S$ is the disconnecting switch;

$U_{\mathrm{L}}$ is the off-load line side voltage;

$\mathrm{M}_{1}$ is bus-bar 1;

$\mathrm{CB}$ is a circuit breaker;

$\mathrm{M}_{2}$ is bus-bar 2;

$\mathrm{W}_{1}$ is the off-load overhead line;

$C_{\mathrm{G}}$ is the stray capacitance of SC;

$\mathrm{Z}_{\mathrm{R}}$ is the equivalent impedance of SCSR;

$\mathrm{Z}_{\mathrm{C}}$ is the equivalent impedance of $\mathrm{SC}$.

The length of $\mathrm{M}_{1}, \mathrm{M}_{2}$, and $\mathrm{W}_{1}$ are $l_{1}, l_{2}$, and $l_{3}$, respectively. $\mathrm{W}_{1}$ is modeled as a short section of overhead transmission line that connects the SCSR and SC in the UHV substation. By using a Laplace transform, $Z_{\mathrm{R}}$ and $Z_{\mathrm{C}}$ are transformed from the time domain into the $s$ domain, as given in the following [27]:

$$
\begin{aligned}
& Z_{\mathrm{R}}(s)=1 / s C_{\mathrm{R}}=1 / s\left(C_{1}+C_{12} / / C_{2}\right), \\
& Z_{\mathrm{C}}(s)=1 / s\left(C_{\mathrm{C}}+C_{\mathrm{D}}+C_{\mathrm{M}}+\left(C_{\mathrm{H}}+C_{\mathrm{S}}\right) / / C_{\mathrm{L}}\right),
\end{aligned}
$$

where $C_{\mathrm{R}}$ is the equivalent capacitance of the SCSR.

Compared with overhead lines, the structure of the GIS bus-bar is much more compact and can be represented by a distributed-parameter model with wave impedance. The wave impedance of the GIS bus-bar and overhead line is [23]

$$
Z_{i}=-j \sqrt{\frac{L_{i}}{C_{i}}} \cot \omega \sqrt{L_{i} C_{i}} l_{n} \quad(i=1,2 ; \quad n=1,2,3),(2)
$$

where $Z_{1}$ is the wave impedance of the GIS bus-bar $\left(M_{1}\right.$ and $\mathrm{M}_{2}$ ), and $Z_{2}$ is the wave impedance of the overhead line $\mathrm{W}_{1} . L_{1}$ is the inductance of the GIS bus-bar and $L_{2}$ is the inductance of the overhead line. $C_{1}$ and $C_{2}$ represent the capacitance of the GIS bus-bar $\left(\mathrm{M}_{1}\right.$ and $\left.\mathrm{M}_{2}\right)$ and the capacitance of the overhead line $\mathrm{W}_{1}$, respectively.

Replacing the cotangent function (2) by a second-order approximation, $Z_{i}(i=1,2)$ can be written in the form [23]

$$
Z_{i} \approx-j\left(\frac{1}{\omega C_{i} l_{n}}-\omega \frac{L_{i}}{3} l_{n}\right) \quad(i=1,2 ; \quad n=1,2,3) \text {. (3) }
$$

The distributed-parameter model of the UHV substation can be further simplified to that shown in Fig. 4.

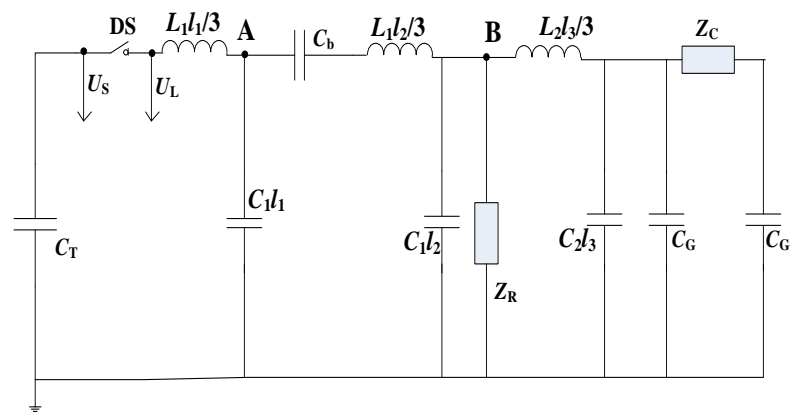

FIG. 4. Equivalent model of ultrahigh voltage station with distributed parameters.

In Fig. $4, C_{\mathrm{b}}$ is the circuit-breaker branch capacitance, and $C_{\mathrm{G}}$ is the SC stray capacitance. The points A and B show the positions of the GIS and SCSR port, respectively. Taking the worst-case scenario into consideration shows that the arc resistance of DS may be ignored. Consequently, the VFTC at both ports of the DS are equal, which is expressed as

$$
\frac{U_{\mathrm{S}}(s)}{Z_{\mathrm{S}}(s)}=-\frac{U_{\mathrm{L}}(s)}{Z_{\mathrm{L}}(s)},
$$

where $U_{\mathrm{S}}(\mathrm{s})$ and $U_{\mathrm{L}}(\mathrm{s})$ are the power supply and off-load line side voltages after Laplace transformation, respectively. Correspondingly, $Z_{\mathrm{S}}(\mathrm{s})$ and $Z_{\mathrm{L}}(\mathrm{s})$ are the power-supply and off-load line side impedances:

$$
\begin{aligned}
& Z_{\mathrm{S}}(s)=1 / s C_{\mathrm{T}}, \\
& Z_{\mathrm{L}}(s)=Z_{\mathrm{A}}(s)+s L_{1} l_{1} / 3 \\
& Z_{\mathrm{A}}(s)=\left(1 / s C_{1} l_{1}\right) / /\left(Z_{\mathrm{B}}(s)+s L_{1} l_{2} / 3+1 / s C_{\mathrm{b}}\right),(5) \\
& Z_{\mathrm{B}}(s)=\left[1 / s\left(C_{\mathrm{R}}+C_{1} l_{2}\right)\right] / / Y_{\mathrm{R}}(s) \\
& Y_{\mathrm{R}}(s)=1 /\left[s\left(C_{\mathrm{G}} / / C_{\mathrm{C}}+C_{2} l_{3}+C_{\mathrm{G}}\right)\right]+s L_{2} l_{3} / 3
\end{aligned}
$$

In Eqs. (5), $Z_{\mathrm{A}}(\mathrm{s})$ and $Z_{\mathrm{B}}(\mathrm{s})$ are the off-load line side 
impedances at $\mathrm{A}$ and $\mathrm{B}$, respectively. Since the angular frequency $\omega$ is not zero, Eq. (4) can be simplified as

$$
Z_{\mathrm{S}}(s)+Z_{\mathrm{L}}(s)=0 \text {. }
$$

The oscillation frequency generated by closing the DS, as denoted by $p_{\mathrm{i}}(i=1, \ldots, n)$, can be obtained by solving Eq. (6). The power-supply-side current $I_{\mathrm{S}}(\mathrm{s})$ of the DS can be expressed as

$$
\begin{gathered}
U_{\mathrm{S}}(s)=\frac{\left(U_{\mathrm{S} 0}-U_{\mathrm{L} 0}\right) Z_{\mathrm{S}}(s)}{s\left[Z_{\mathrm{S}}(s)+Z_{\mathrm{L}}(s)\right]}=\frac{N_{\mathrm{S}}(s)}{D_{\mathrm{S}}(s)}=\sum_{i=1}^{n} \frac{n_{1, i}}{s-p_{i}}, \\
I_{\mathrm{S}}(s)=\frac{\left(U_{\mathrm{S} 0}-U_{\mathrm{L} 0}\right)}{s\left[Z_{\mathrm{S}}(s)+Z_{\mathrm{L}}(s)\right]}=\frac{M_{\mathrm{S}}(s)}{D_{\mathrm{S}}(s)}=\sum_{i=1}^{n} \frac{m_{1, i}}{s-p_{i}},
\end{gathered}
$$

where $U_{\mathrm{S} 0}$ and $U_{\mathrm{L} 0}$ are the residual voltages of the power-supply side and off-load line side before closing the DS, respectively. $N_{\mathrm{S}}(\mathrm{s})$ and $D_{\mathrm{S}}(\mathrm{s})$ are the numerator and denominator of $U_{\mathrm{S}}(\mathrm{s}) . M_{\mathrm{S}}(\mathrm{s})$ is the numerator of $I_{\mathrm{S}}(s) . n_{1, \mathrm{i}}(i=$ $1, \ldots, \mathrm{n})$ and $m_{1, \mathrm{i}}(i=1, \ldots, n)$ are coefficients, which can be obtained by

$$
\begin{aligned}
& n_{1, \mathrm{i}}=\lim _{s \rightarrow p_{i}} \frac{\left(s-p_{i}\right) N_{\mathrm{S}}(s)}{D_{\mathrm{S}}(s)}=\frac{N_{\mathrm{S}}\left(p_{i}\right)}{D_{\mathrm{S}}^{\prime}\left(p_{i}\right)}, \\
& m_{1, \mathrm{i}}=\lim _{s \rightarrow p_{i}} \frac{\left(s-p_{i}\right) M_{\mathrm{S}}(s)}{D_{\mathrm{S}}(s)}=\frac{M_{\mathrm{S}}\left(p_{i}\right)}{D_{\mathrm{S}}{ }^{\prime}\left(p_{i}\right)}, \quad(i=1,2, \ldots, n) .
\end{aligned}
$$

Thus, $U_{\mathrm{S}}(t)$ and $I_{\mathrm{S}}(t)$ in the time domain can be expressed as

$$
\begin{aligned}
U_{\mathrm{S}}(t) & =\sum_{i=1}^{n} n_{1, \mathrm{i}} e^{p_{i} t}=\sum_{i=1}^{n} \frac{N_{\mathrm{S}}\left(p_{i}\right)}{D_{\mathrm{S}}{ }^{\prime}\left(p_{i}\right)} e^{p_{i} t} \\
I_{\mathrm{S}}(t) & =\sum_{i=1}^{n} m_{1, \mathrm{i}} e^{p_{i} t}=\sum_{i=1}^{n} \frac{M_{\mathrm{S}}\left(p_{i}\right)}{D_{\mathrm{S}}{ }^{\prime}\left(p_{i}\right)} e^{p_{i} t}
\end{aligned}
$$

Similarly, $U_{\mathrm{A}}(s)$ and $I_{\mathrm{A}}(s)$ can be expressed as

$$
\begin{aligned}
& U_{\mathrm{A}}(s)=\frac{U_{\mathrm{S}}(s) Z_{\mathrm{A}}(s)}{Z_{\mathrm{L}}(s)+Z_{\mathrm{A}}(s)}=\frac{N_{\mathrm{A}}(s)}{D_{1}(s)}=\sum_{i=1}^{n} \frac{n_{2, i}}{s-p_{i}}, \\
& I_{C_{\mathrm{b}}}(s)=\frac{U_{\mathrm{A}}(s)}{Z_{\mathrm{B}}(s)+s L_{1} l_{2} / 3+1 / s C_{\mathrm{b}}} .
\end{aligned}
$$

Finally, $U_{\mathrm{B}}(s)$ and $I_{\mathrm{B}}(s)$ are

$$
\begin{aligned}
& U_{\mathrm{B}}(s)=U_{\mathrm{A}}(s) \frac{Z_{\mathrm{B}}(s)}{Z_{\mathrm{B}}(s)+s L_{1} l_{2} / 3+1 / s C_{\mathrm{b}}}, \\
& I_{\mathrm{R}}(s)=U_{\mathrm{B}}(s) s C_{\mathrm{R}} .
\end{aligned}
$$

For UHV power systems in China, the typical parameters of UHV substations are $U_{\mathrm{S}}=1100 / \sqrt{3} \mathrm{kV}, Z_{1}=90 \Omega, Z_{2}=500 \Omega$, $l_{1}=10 \mathrm{~m}, l_{2}=50 \mathrm{~m}, l_{3}=100 \mathrm{~m}, C_{\mathrm{T}}=10 \mathrm{nF}, c=300 \mathrm{~m} / \mathrm{s}, C_{\mathrm{b}}=$ $600 \mathrm{pF}, C_{\mathrm{R}}=500 \mathrm{pF}$, and $C_{\mathrm{C}}=1 \mu \mathrm{F}$. The VFTC waveform in the SCSR is computed by using the aforementioned method. Meanwhile, the model is established in the environment of the EMTP. Figure 5 shows that the calculated and simulated waveforms are consistent with each other. The computational error between calculation and simulation is quite small and can essentially be neglected. However, compared with the simulation, the VFTC characteristics can be obtained more rapidly by using the proposed calculation. Thus, the proposed method to calculate the VFTC is feasible and saves time. Applying a Fourier transform shows that the frequency of the VFTC waveform in the SCSR ranges from $100 \mathrm{kHz}$ to $50 \mathrm{MHz}$.

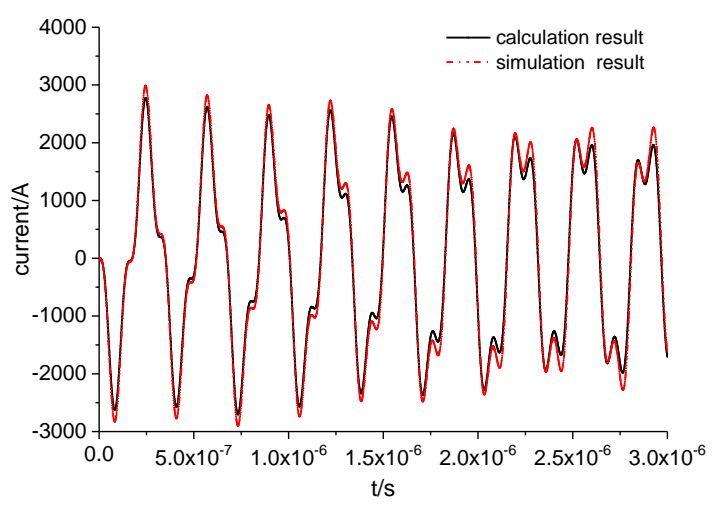

FIG. 5. Comparison of calculated and simulated VFTC waveforms in the stepped controlled shunt reactor.

\section{Case Study}

4.1 Very fast transient current generated by the switching operation of disconnecting switch in gas-insulated switchgear

The VFTC is be generated upon activating the DS in the GIS. At the same time, the VFTC flows through the circuit breaker and the SCSR. As mentioned above, the main oscillation frequency and the corresponding amplitude are correctly calculated by using the analytical method. The results are listed in Table I.

TABLE I. Amplitude and frequency of very fast transient current in breaker and controlled reactor in ultra high-voltage power system

\begin{tabular}{ccc}
\hline Location in station & $\begin{array}{c}\text { Amplitude of VFTC } \\
\text { component }(\mathrm{kA})\end{array}$ & $\begin{array}{c}\text { Oscillation frequency } \\
(\mathrm{MHz})\end{array}$ \\
\hline & $A_{1}=0.2336$ & \\
$A_{2}=9.66$ & $f_{1}=0.92$ \\
Breaker $\left(C_{\mathrm{b}}\right)$ & $A_{3}=2.828$ & $f_{2}=3.03$ \\
& $A_{1}=0.0928$ & $f_{3}=9.32$ \\
& $A_{2}=1.785$ & \\
\hline \multirow{2}{*}{ Controlled reactor } & $A_{3}=0.603$ & \\
$\left(\mathrm{Z}_{\mathrm{R}}\right)$ &
\end{tabular}

The elements including $f_{1}, f_{2}$, and $f_{3}$ in the third column of Table I are the frequencies of the three different main components of VFTC. The elements in the second column are the corresponding amplitudes of the main components of the VFTC at the above three frequencies. For example, from the Fourier-analysis results of the VFTC in the circuit breaker, the amplitudes of the main VFTC frequency components of 0.92 , 3.03, and $9.32 \mathrm{MHz}$ are 0.2336, 9.66, and $2.828 \mathrm{kA}$, respectively.

According to Table I, the main frequency components of the VFTC in the circuit breaker and controlled shunt reactor are $f_{2}$ and $f_{3}$. The impedance $Z_{\mathrm{C}}$ of the SC capacitor is relatively large and the approximations will not significantly affect the VFTC. Thus, the impedance $Z_{C}$ of the SC capacitor may be considered as a short circuit under the action of the very fast transient wave. Figure 6 shows how the SCSR affects the main frequency component of the VFTC. 


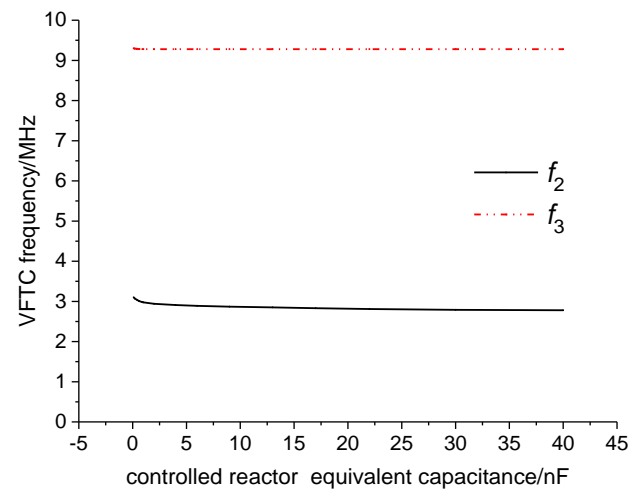

FIG. 6. Frequency of very fast transient current as a function of equivalent capacitance $C_{\mathrm{R}}$ of controlled reactor.

Figure 6 shows that $f_{3}$ is nearly constant with increasing $C_{\mathrm{R}}$. However, $f_{2}$ drops from 3.22 to $2.81 \mathrm{MHz}$. As $C_{\mathrm{R}}$ changes from 0.1 to $8 \mathrm{nF}$, the frequency varies more than it does when $C_{\mathrm{R}}$ changes from 8 to $40 \mathrm{nF}$. The value of $C_{\mathrm{R}}$ can be changed by adjusting the degree of compensation of the SCSR. Therefore, the frequency of the VFTC can be tuned by adjusting the compensation degree of the SCSR.

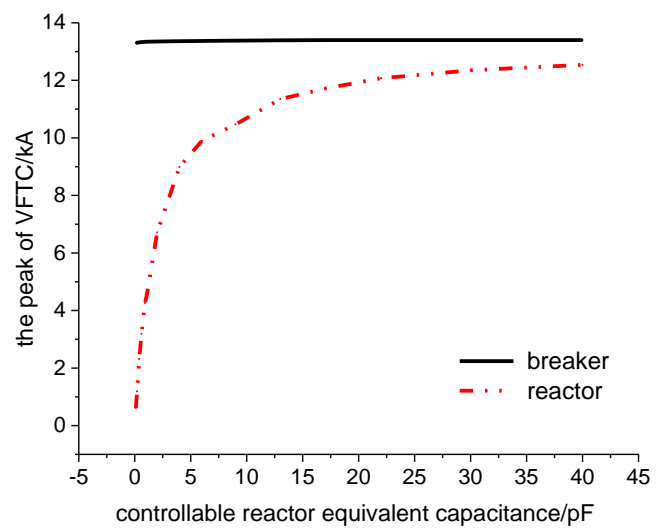

FIG. 7. Peak value of very fast transient current as a function equivalent capacitance $C_{\mathrm{R}}$ of controlled reactor.

The VFTC frequency as a function of equivalent capacitance of the controlled reactor differs significantly from the peak of VFTC in the circuit breaker as a function of $C_{\mathrm{R}}$ of the controlled reactor (see Fig. 7). Clearly, the peak of VFTC in the circuit breaker is almost independent of $C_{\mathrm{R}}$ and is equal to $13.2 \mathrm{kA}$. However, the peak of VFTC is remarkably nonlinear in $C_{\mathrm{R}}$ in the controlled reactor (see red curve in Fig. 7). When $C_{\mathrm{R}}=0.1$ $\mathrm{nF}$, the peak of VFTC in the controlled reactor is $0.9 \mathrm{kA}$, which increases to $12.6 \mathrm{kA}$ as $C_{\mathrm{R}}$ increases. Note that the slope of the curve decreases. When $C_{\mathrm{R}}$ rises to $30 \mathrm{nF}$, the curve is nearly horizontal. These results indicate that the peak of VFTC in the circuit breaker is independent of $C_{\mathrm{R}}$ whereas the peak of VFTC in the controlled reactor increases with $C_{\mathrm{R}}$.

According to Fig. 4, the VFTC in the circuit breaker is the sum of those in the other branches, so the peak of VFTC in the circuit breaker is greater than that in the controlled reactor in Fig. 7. Because the stray capacitance of the controlled reactor is much less than the capacitance to ground of the transmission line, the voltage of point $\mathrm{B}$ changes little when the stray capacitance of the controlled reactor changes from 0 to $45 \mathrm{nF}$. As a result, upon increasing the equivalent capacitance of the controlled reactor, the VFTC in the circuit breaker remains almost constant and the VFTC in the controlled reactor increases rapidly. This explains why the curves of VFTC as a function of $C_{\mathrm{R}}$ in circuit breaker and in the controlled reactor differ so dramatically.

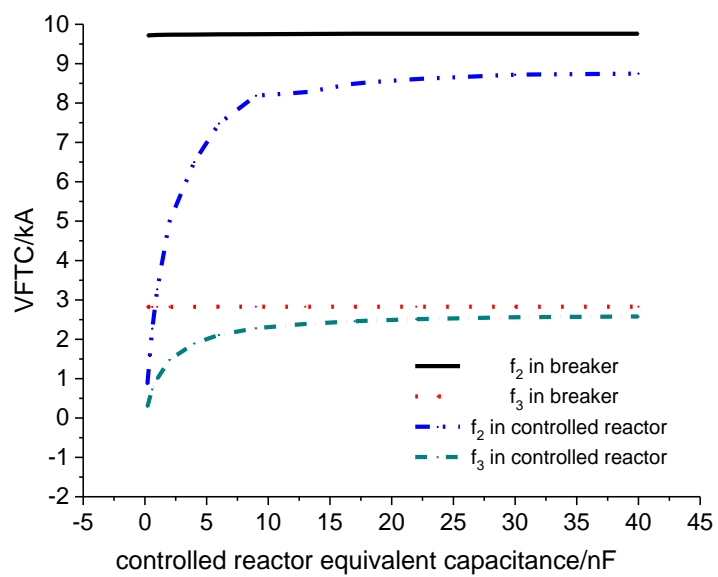

FIG. 8. Amplitude of frequency components of very fast transient current as a function of equivalent capacitance $C_{\mathrm{R}}$ of controlled reactor.

The amplitude-frequency characteristic of the VFTC is the main factor that determines the design of the internal insulation of the circuit breaker and the SCSR. Thus, the amplitude of the frequency components of the VFTC as a function of $C_{\mathrm{R}}$ need to be further studied. Figure 8 shows these curves for the circuit breaker and the SCSR.

In Fig. 8 , the amplitudes of frequency components $f_{2}$ and $f_{3}$ of the VFTC in the breaker are almost independent of $C_{R}$. Conversely, those for the VFTC in the SCSR increase as $C_{\mathrm{R}}$ increases. Specifically, when $C_{\mathrm{R}}=0.1 \mathrm{nF}$, the amplitudes of frequencies $f_{2}$ and $f_{3}$ in the SCSR are minimal and the slope of the curve is steepest. For $C_{\mathrm{R}}=15 \mathrm{nF}$, the amplitudes of frequencies $f_{2}$ and $f_{3}$ in the SCSR approach to the maximum. The amplitudes corresponding to $f_{2}$ and $f_{3}$ are 8.5 and $2.5 \mathrm{kA}$, respectively. These results show that the amplitude of the main frequency components of the VFTC in the SCSR depend on $C_{\mathrm{R}}$.

\subsection{Very fast transient current generated by the operation of disconnecting switch in series compensation}

Switching the DS in the SC platform can cause multiple arcs. With the influence of SC stray capacitor, a VFTC will be generated in the SCSR, which would threaten the internal insulation of the SCSR. Figure 9 shows the simplified equivalent model of the substation when the DS in the SC switches on, where M12 is the bus-bar.

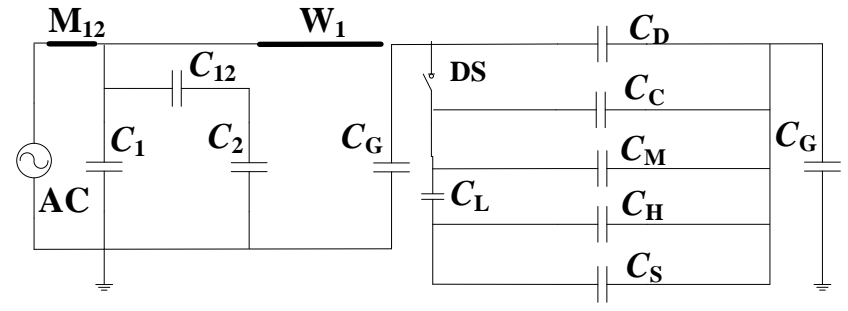

FIG. 9. Simplified model of ultra high-voltage substation during operation of disconnecting switch in series compensation. 
When the DS in SC closes, the amplitude and frequency characteristics of the VFTC in the SCSR depend mainly on the SC stray capacitor $C_{\mathrm{G}}$ and the length of overhead line $\mathrm{W}_{1}$. Applying a Laplace transform gives the amplitude-frequency characteristics of the VFTC in the SCSR as a function of $C_{\mathrm{G}}$ and $\mathrm{W}_{1}$ (see results in Figs. 10 and 11).

As $C_{\mathrm{G}}$ increases from 0.1 to $40 \mathrm{nF}$ (Fig. 10), the frequency of $f_{01}$ of the VFTC in the SCSR decreases from 3.8 to $3.0 \mathrm{MHz}$, the frequency $f_{02}$ decreases from 1.93 to $0.98 \mathrm{MHz}$, and the peak increases from 0.61 to $3.57 \mathrm{kA}$. When $C_{\mathrm{G}}$ exceeds $5 \mathrm{nF}$, the peak VFTC remains constant in the SCSR. These results indicate that, although the VFTC frequency in the SCSR decreases with increasing SC stray capacitance, the frequency $f_{01}$ and the peak VFTC remain quite large, which would threaten the operation of the SCSR. Thus, the SC stray capacitance must be further reduced.

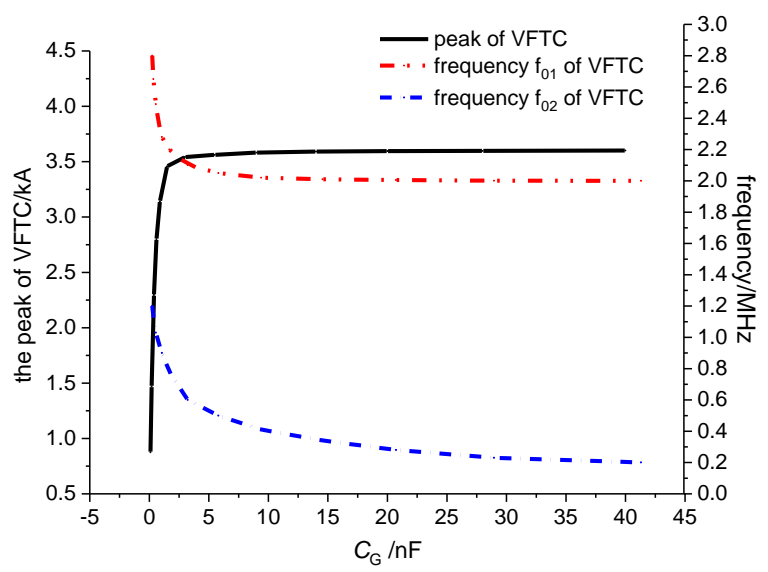

FIG. 10. Peak of VFTC and frequency of two frequency components of VFTC in controlled reactor as a function of $\mathrm{C}_{\mathrm{G}}$.

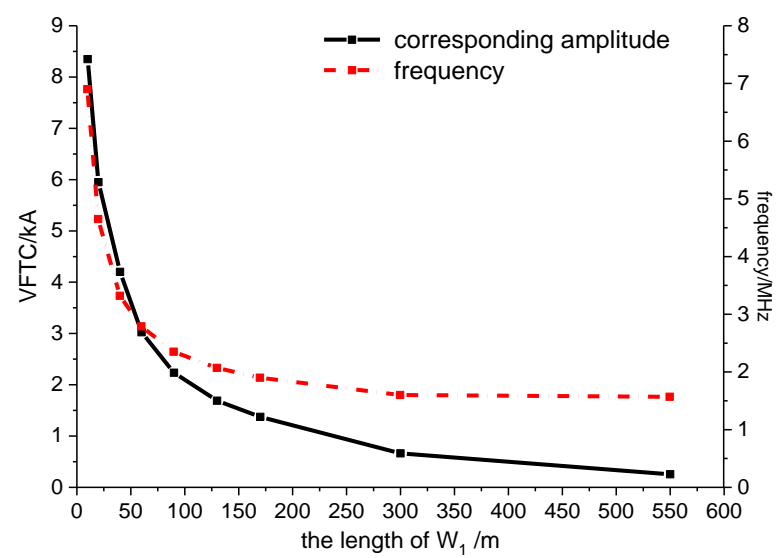

FIG. 11. VFTC amplitude and frequency in controlled reactor as a function of length of $\mathrm{W}_{1}$.

As the length of $\mathrm{W}_{1}$ increases from 10 to $550 \mathrm{~m}$ (Fig. 11), the main frequency of VFTC decreases from 7.75 to $1.6 \mathrm{MHz}$. Meanwhile, the corresponding amplitude of VFTC in the SCSR drops from 8.47 to $0.22 \mathrm{kA}$. The lengthening of the overhead line between SC and the SCSR can effectively suppress the VFTC in the SCSR.

\section{CONCLUSIONS}

The technique of HRPC can solve the new problems resulting from the rapid growth of transmission power and the dynamic variations in reactive power. As such, this technique may find widespread application in the design of the UHV power grid. Because of high voltage and the bulk power capacity of GIS UHV substations, the VFTC in UHV substations will present new features with HRPC. However, the characteristics of frequency components of VFTC have not been studied for UHV systems with HRPC. The present work addresses this void by studying this relationship as a function of various system parameters.

The time-domain VFTC is deduced by using an inverse Laplace transform for a SCSR and a SC in a UHV substation with HRPC. The theoretical analysis presented herein of the characteristics of the VFTC at different locations and for various scenarios of disconnecting switching should provide a theoretical basis of analysis for the power industry. Based on the results of these calculations, we draw the following conclusions:

(1) The VFTC consists of various sinusoidal waves of differing frequencies, the amplitude of which depends on the capacitance of the equipment and the line length. The frequency of the main component is significantly affected by the locations of VFTC in the substation, and the peak of VFTC in the GIS is greater than that in the SCSR.

(2) When the DS of the GIS is closed, the frequency of the VFTC in the SCSR can be tuned by adjusting the compensation degree of the SCSR. The peak of VFTC in the circuit breaker is independent of $C_{R}$. However, the peak of VFTC and the amplitude of the main frequency component of the VFTC in the SCSR both depend on $C_{\mathrm{R}}$.

(3) When DS in SC is closed, the VFTC oscillation frequencies in the SCSR decrease with increasing SC stray capacitance. However, despite this, the frequencies and peak of VFTC remain large. Increasing the length of the overhead line between the SC and the SCSR with HRPC can suppress the VFTC in the SCSR.

\section{Acknowledgement}

This work is jointly supported by Shandong Provincial Science Foundation of China (BS2015NJ011), Key Research and Development Program of Shandong Province (2018GGX103048), and National Natural Science Foundation of China (51507095).

\section{REFERENCES}

[1] Lei AY, Dong XZ, Shi SX, Wang B, Terzija V. Equivalent traveling waves based current differential protection of EHV/ UHV transmission lines. Int J Electr Power Energy Syst 2018; 97: 282-289.

[2] Dong XZ, Luo SX, Shi SX, Wang B, Wang SY, Ren L, et al. Implementation and application of practical traveling-wave-based directional protection in UHV transmission lines. IEEE Trans. Power Del. 2016; 31(1): 294-302. 
[3] Li Y, He JL, Yuan J, Li C, Hu J, Zeng R. Failure risk of UHV AC transmission line considering the statistical characteristics of switching overvoltage wave shape. IEEE Trans. Power Del. 2013; 28(3): 1731-1739.

[4] Helmut H. Testing of reactor switching for UHV circuit breakers. IEEE Trans. Power Del. 2015; 30(3): 1172-1178.

[5] Kang N, Chen JX, Liao Y. A fault-location algorithm for series-compensated double-circuit transmission lines using the distributed parameter line model. IEEE Trans. Power Del. 2015; 30(1): 360-367.

[6] Yang G, Li L, Zhang XL, Jia Q, Liu LQ. A transient model for controlled shunt reactor based on duality theory. IEEE Trans. Mag. 2015; 51(3): 310-313.

[7] Biswal M, Pati BB, Pradhan AK. Adaptive distance relay setting for series compensated line. Int J Electr Power Energy Syst 2013; 52 (2013): 198-206.

[8] N. Kirthika, S. Balamurugan. A new dynamic control strategy for power transmission congestion management using series compensation. Int J Electr Power Energy Syst 2016; 77(2016): 271-279.

[9] Finotti C, Gaio E. Continuous model in dq frame of Thyristor Controlled Reactors for stability analysis of high power electrical systems. Int J Electr Power Energy Syst 2014; 63 (2014): 836-845.

[10] Li RC, Liu HS, Lou J, Wang Z, Li B, Ji L. Influence of ultra-high-voltage hybrid reactive power compensation on the interrupting characteristics of $\mathrm{CB}$ at out-of-phase faults. IET Generation, Transmission \& Distribution 2017; 11(5): 1258-1264.

[11] Rao MM, Thomas MJ, Singh BP. Electromagnetic Field Emission From Gas-to-Air Bushing in a GIS During Switching Operations. IEEE Transactions on Electromagnetic Compatibility 2007; 49(2): 313-321.

[12] Zhan HM, Duan SF, Li CR, Yao LZ, Zhao L. A Novel Arc Model for Very Fast Transient Overvoltage Simulation in a 252-kV Gas-Insulated Switchgear. IEEE Trans. Plasma Sci. 2014; 42(10): 3423-3429.

[13] Rao MM, Thomas MJ, Singh BP. Frequency characteristics of very fast transient currents in a 245-kV GIS. IEEE Trans. Power Del. 2005; 20(4): 2450-2457.

[14] M. M. Rao, M. J. Thomas and B. P. Singh. Shielding Effectiveness of the Gas-Insulated Bus Duct for Transient EM Fields Generated in a GIS During Switching Operations. IEEE Trans. Power Del. 2008; 23(4): 1946-1953.

[15] Li C, He JL, Hu J, Zeng R, Yuan J. Switching transient of 1000-kV UHV system considering detailed substation structure. IEEE Trans. Power Del 2012; 27(1): 112-122.
[16] Tavakoli A, Gholami A, Parizad A, Soheilipour HM, Nouri H. Effective factors on the very fast transient currents and voltage in the GIS. Proc. IEEE Transmission and Distribution Conference and Exposition: Asia and Pacific. 2009.

[17] Liu JM, Wei Du, Wang ZZ, Yi Sun, Li YG, Fang XC. A new simulation calculation method for VFTO based on time domain finite element method. Trans. China Electrotechnical Society 2013; 28(11): 286-292.

[18] Xu JY, Si BG, Lin X, Lu L. Electric field parameter calculation of extra-high voltage GIS disconnector based on dummy dielectric constant method. Trans. China Electro technical Society 2008; 23(5): 37-42.

[19] Insulation coordination for UHV AC Systems. CIGRE TB306, 2011.

[20] Zhang L, Mao C, Pu L, et al. Conical voltage sensor for measuring very fast transient overvoltage up to $3 \mathrm{MV}$ in ultra-high-voltage class gas-insulated switchgear. IET Science, Measurement \& Technology 2018; 12(3): 405-410.

[21] Tavakoli A, Gholami A, Nouri H, Negnevitsky M. Comparison between suppressing approaches of very fast transients in gas-insulated substations (GIS). IEEE Trans. Power Del. 2013; 28(1): 303-310.

[22] Shao C, Yang Y, Wang ZJ. Modeling of GIS switching arc and its effect on VFTO waveforms. Power System Technology 2010; 34(7): 200-205.

[23] Li QM, Wu ML. Simulation method for the applications of ferromagnetic materials in suppressing high-frequency transients within GIS. IEEE Trans. Power Del. 2007; 22(3): 1628-1632.

[24] Gajare S, Pradhan AK, and. Brahma Sukumar M. Model verification of fixed series compensation devices using synchronized data. IEEE Trans. Power Del. 2016; 31(1): 174-181.

[25] Saha MM, Kasztenny B, Rosolowski E, Izykowski J. First zone algorithm for protection of series compensated lines. IEEE Trans. Power Del. 2001; 16(2): 200-207.

[26] Zheng WJ. Stability effects research of stepped controlled shunt reactor inner faults to power grid. Proc. China International Conference on Electricity Distribution. 2012.

[27] Povh D, Schmitt H, Volcker O, Wltzmann R. Modeling and analysis guidelines for very fast transients. IEEE Trans. Power Del. 1996; 11(4): 2028-2035. 